\section{El debate en América Latina sobre la participación de los hombres en programas de salud reproductiva}

\author{
Martine Maria Adriana \\ de Schutter ${ }^{1}$
}

1 Organización Panamericana de la Salud, Programa sobre Mujer, Salud y Desarrollo y Programa sobre Salud Familiar y Población. Dirección postal: 525, 23rd Street N.W. Washington, D.C. 20037, Estados Unidos de América. Teléfono: 202 974-3468. Fax: 202 974-3671. Correo electrónico: schuttem@paho.org
A raíz de la Conferencia Internacional de Población y Desarrollo (El Cairo, 1994) y de la Cuarta Conferencia Mundial de la Mujer (Beijing, 1995), el tema de la participación de los hombres en los programas de salud reproductiva ha despertado mayor interés entre entidades tan diversas como los planificadores y proveedores de servicios de salud; las agencias de cooperación; los movimientos para la defensa de los derechos de la mujer; las instituciones académicas, y los grupos de reflexión integrados por hombres. Este interés, cada vez más marcado, se debe principalmente al reconocimiento de que los hombres desempeñan un papel importante en la toma de decisiones de la pareja sobre la sexualidad y la reproducción y a la conciencia de que la falta de equidad en las relaciones entre hombres y mujeres menoscaba la salud reproductiva de ambos sexos. Existen, además, pruebas cada vez más patentes de que la conducta sexual de algunos hombres pone en peligro la salud de la mujer y de los hijos. Por último, el interés obedece también al deseo de prevenir los embarazos no deseados o anticipados mediante el uso de métodos de planificación familiar; a la solicitud por parte de las propias mujeres de que sus parejas participen en las actividades de promoción, educación y provisión de servicios; a la preocupación por la alta frecuencia de las infecciones de transmisión sexual (ITS), incluida la infección por $\mathrm{VIH} ; \mathrm{y}$, al deseo manifestado por un creciente número de hombres de participar en actividades y programas destinados a mejorar su propia salud reproductiva y la de su familia y pareja sexual (1).

En las últimas décadas los indicadores del estado de salud sexual y reproductiva en la Región de las Américas han mejorado notablemente. No obstante, los adelantos no han sido uniformes y sigue habiendo indicadores de salud inaceptables. Se observan cifras elevadas de embarazos no planificados o deseados en la adolescencia; de ITS, incluida la infección por VIH; de morbilidad y mortalidad maternas; de cáncer cervicouterino; de abortos inducidos con complicaciones, y de violencia sexual y doméstica (2). A estos indicadores alarmantes contribuye el hecho de que las actividades de prevención, promoción y atención en el ámbito de la salud reproductiva raras veces se dirigen a los hombres.

Muchos reconocen la importancia de incorporar a la población masculina en los programas de salud reproductiva, pero no todos coinciden sobre el carácter que debe tener esa incorporación. En el 
presente artículo se resumen y examinan algunos de los temas que forman parte del debate actual en América Latina en torno a la participación de los hombres en dichos programas.

\section{OBJETIVOS DE INCLUIR A LOS HOMBRES EN LOS PROGRAMAS DE SALUD REPRODUCTIVA}

Aunque en general se reconoce la necesidad de que los hombres participen en las iniciativas para la promoción de la salud reproductiva, hay diferencias de opinión en cuanto al objetivo principal de esta participación. Para algunos, el objetivo consiste en atender a las necesidades particulares de los hombres en el campo de la salud reproductiva; para otros, en fomentar, con la ayuda de los hombres, la salud reproductiva de la mujer.

Muchas organizaciones y movimientos sociales para la defensa de los intereses de la mujer conceden a los hombres un papel central en el esfuerzo por mejorar la salud reproductiva de las mujeres. Sus argumentos se reducen a tres fundamentalmente: 1) que las mujeres sufren mayor morbilidad y mortalidad por causas reproductivas que los hombres; 2) que aquellas tienen una mayor responsabilidad, por razones fisiológicas y sociales, en asuntos relacionados con la fertilidad, el embarazo y la crianza de los hijos; y 3) que todavía no se han resuelto los problemas relacionados con la accesibilidad, cobertura y calidad de los servicios de salud reproductiva destinados a las mujeres $(3,4)$.

Este punto de vista se ve respaldado por algunos datos de la OMS para América Latina, según los cuales los años de vida ajustados en función de la discapacidad (DALY) por razones vinculadas con la sexualidad y la reproducción representan $16,8 \%$ del total entre las mujeres y $4,25 \%$ entre los hombres de 15 a 44 años de edad. Estas cifras abarcan las ITS, incluida la infección por VIH, así como todas las causas de morbilidad materna y los distintos cánceres del aparato reproductor (5).

La mayor atención que siempre se ha prestado a la salud reproductiva de la mujer ha resultado en una ausencia de información sobre las necesidades de los hombres en este campo y sobre la influencia del hombre en la salud reproductiva de la mujer. De hecho, la población masculina se ha visto incluida en las Encuestas de Demografía y Salud (DHS) solamente en los últimos años, aunque son alentadoras la cantidad, calidad y variedad de la información que se ha generado últimamente sobre el tema dentro y fuera de la Región (6-9).

Si bien es indudable que las mujeres tienen una mayor carga de problemas relacionados con la función reproductora, los hombres también tienen necesidades en este ámbito que exigen atención. Muchos temen, sin embargo, que se produzca una competencia entre los recursos e iniciativas dedicados a la salud reproductiva de las mujeres y a las de los hombres. Varias entidades para la defensa de los intereses de la mujer, organizaciones no gubernamentales (ONG) y agencias de cooperación dudan de la conveniencia de llevar a cabo iniciativas para la atención exclusiva de las necesidades de los hombres -financiar, por ejemplo, servicios de cáncer de próstata o la cobertura de medicamentos como Viagra $^{\circledR-}$, porque no necesariamente tienen un impacto favorable en la salud de las mujeres. En una reunión latinoamericana de ONG que fue auspiciada parcialmente por la OPS en 1997 en Quito, Ecuador, y cuyos temas centrales fueron la salud y los derechos sexuales y reproductivos, se señaló que ". . . dentro del movimiento de salud de las mujeres existe la preocupación de que, al enfocar más la salud reproductiva en relación a la masculinidad, se desviarán los recursos financieros y humanos destinados a la salud sexual y reproductiva de las mujeres" (4).

La OPS tiene el mandato de promover la equidad en el campo de la salud, entendiendo por inequidad la presencia de "... diferencias en salud que no sólo son innecesarias y evitables sino que, además, se consideran incorrectas e injustas" (10). Atendiendo a esta definición, la OPS ha identificado a los hombres como población subatendida y ha lanzado una iniciativa para promover su participación en programas de salud reproductiva en Centroamérica (2). ${ }^{2}$ Habiéndose reconocido que ambos sexos tienen necesidades válidas, es menester, en el contexto de procesos de reforma del sector de la salud con presupuestos limitados, balancear los intereses de ambos sexos y tomar medidas para satisfacerlas según su prioridad.

\section{LA MASCULINIDAD HEGEMÓNICA Y LA EQUIDAD DE GÉNERO}

Otro debate actual gravita en torno a la conveniencia y factibilidad de promover la participación de los hombres en programas de salud reproductiva sin meditar en el impacto negativo que podrían tener la construcción social denominada "masculi-

\footnotetext{
Actualmente, la OPS colabora con la subregión Centroamericana para desarrollar estrategias innovadoras que promuevan la participación de los hombres en los programas de salud reproductiva con conciencia del género. El proyecto cuenta con el auspicio del Gobierno de la República Federal de Alemania a través de la Agencia Técnica Alemana (GTZ) y se realiza en Belice, Costa Rica, El Salvador, Guatemala, Honduras, Nicaragua y Panamá.
} 
nidad hegemónica" ${ }^{3}$ y las inequidades de género ${ }^{4}$ en el campo de la salud reproductiva. Muchas entidades, entre ellas la mayoría de los grupos de reflexión integrados por hombres en la Región, consideran que promover la participación de los hombres en programas de salud reproductiva supone cimentar en ellos nuevas formas de ser y pensar, es decir, una mentalidad equitativa y democrática en torno a lo masculino y feminino. Pero otras, entre ellas varios gerentes y proveedores de servicios de salud reproductiva, ponen en tela de juicio, aduciendo diversas razones, la conveniencia de orientar los servicios de salud pública hacia la búsqueda de un nuevo concepto de "lo masculino". Algunos consideran que el asunto es muy complejo, que cualquier cambio se produce a largo plazo, y prefieren concentrarse en aspectos más concretos que mejoran la salud reproductiva de hombres y mujeres de manera patente en un plazo más corto. Otros reconocen la necesidad de lograr cambios profundos en el comportamiento de los hombres, pero señalan que los recursos son limitados y que es necesario dar prioridad a algunas necesidades concretas que aún no han sido satisfechas. También se debate si el sector de la salud es el más adecuado para tratar un asunto tan complejo como el de la masculinidad, teniendo en cuenta que los servicios y programas de salud reproductiva no cuentan con personal capacitado, recursos financieros, tiempo suficiente o instalaciones adecuadas para ello. Y finalmente, hay quienes consideran innecesario cambiar las relaciones de género actuales, puesto que no las consideran inadecuadas $(1,14-17)$.

En la Región se han emprendido, por un lado, intervenciones de orden práctico e inmediato, como las destinadas al control del cáncer de próstata, que

\footnotetext{
3 El término masculinidades es interpretado por Connell (11) como el conjunto de configuraciones y prácticas estructuradas por las relaciones de género, que son inherentemente históricas y cuya construcción y reconstrucción es un proceso político que afecta al balance de intereses en la sociedad y la dirección del cambio social. Al ser social y culturalmente construida, no existe una forma única de masculinidad sino diversas masculinidades, que marcan la vida, la salud y enfermedad del hombre según su estrato socioeconómico, origen étnico, cultura, identidad sexual, edad, y otros factores. Aunque hay muchas formas de ser hombre, algunas son socialmente o culturalmente más valoradas que otras y los hombres se sienten presionados a consolidarse en función de esas exigencias propias de lo que es considerada la masculinidad hegemónica, que ". . . se puede definir como la configuración de práctica genérica que encarna la respuesta corrientemente aceptada al problema de la legitimidad del patriarcado, la que garantiza (o se toma para garantizar) la posición dominante de los hombres y la subordinación de las mujeres" (12).

4 La socialización diferenciada de hombres y mujeres conlleva identidades, responsabilidades, funciones y poderes de género diferentes. La perspectiva de género analiza la dinámica de las relaciones entre los sexos para evaluar el impacto que tiene esas relaciones sobre la salud, la posición y los derechos de la mujer y del hombre, incorporando en su análisis las diferencias y especificidades biológicas de ambos, como también la interacción entre factores biológicos y sociales. (13)
}

tratan de responder principalmente a las necesidades biológicas de los hombres o de mejorar la efectividad de los programas de planificación familiar, dejando a un margen la falta de equidad en las relaciones de género. Por otro lado, algunos grupos y organizaciones promueven la reflexión y actividades de autoayuda encaminadas a combatir la identidad masculina hegemónica. ${ }^{5}$ Todo ello implica una labor cuyos efectos se observan en el largo plazo y que acarrea profundos cambios personales y sociales.

En 1998 se celebró en Oaxaca, México, el simposio latinoamericano "Participación masculina en la salud sexual y reproductiva: nuevos paradigmas", que contó entre sus participantes a proveedores de servicios de salud, funcionarios públicos, representantes de movimientos y grupos de hombres y mujeres y de ONG y agencias de cooperación de la Región. En dicho evento se llegó a la conclusión de que los programas de salud reproductiva no deben limitarse a proveer atención clínica, sino que deben ayudar a los hombres a identificar cómo su identidad masculina y las percepciones que tienen en torno a ella influyen en su conducta en relación con la sexualidad, la violencia, la prevención de las ITS y la paternidad (15).

Quienes proponen promover la participación de los hombres en los programas de salud reproductiva argumentan que es peligroso hacerlo sin combatir la masculinidad hegemónica, ya que ello podría resultar en lo que Leñero (18) denomina el "neomachismo". Aunque a primera vista se aprecian ciertos cambios en la actitud masculina con respecto a la equidad de género, en respuesta a los nuevos papeles que ha asumido la mujer, en el fondo los hombres siguen pensando y obrando con actitudes propias de la masculinidad hegemónica $(16,18)$. Según Keijzer, "estos hombres, instalados en una especie de 'machismo light' ya no ejercen (o ya no pueden ejercer) el patriarcado como lo hicieron sus padres y abuelos: ya negocian más las decisiones, ya 'permiten' o 'aceptan' que trabaje la mujer fuera de casa (de preferencia sin ganar más que ellos), pero mantienen un marco de referencia con un claro encuadre machista" (19).

Si se toma la equidad de género como principio básico para justificar la participación de los hombres, siguiendo el mandato de las conferencias de El Cairo y Beijing, no es posible obtener resultados sostenibles en la labor con la población masculina sin combatir el concepto hegemónico de la masculinidad y reemplazarlo por un concepto democrático y equitativo. Para lograrlo, los progra-

\footnotetext{
5 Como, por ejemplo, CORIAC (México), Colectivo Hombres sin Máscara (Honduras), Puntos de Encuentro (Nicaragua), Salud y Género (México), ECOS (Brasil).
} 
mas públicos de salud reproductiva deberán buscar un equilibrio entre la atención a las necesidades de orden práctico de los hombres y el logro de cambios sociales en el mediano y largo plazo. No es fácil introducir los conceptos de masculinidad y feminidad en el contexto de las normas de atención, indicadores de salud y actividades del sector de la salud, y la complejidad del proceso puede desanimar a muchos. Por otro lado, la falta de suficientes pruebas de la eficacia de estas estrategias llevan a otros a realizar sus actividades según los marcos ya establecidos.

Para facilitar la búsqueda de un equilibrio entre la atención de las necesidades directas de los hombres y de las mujeres y la toma de medidas estratégicas encaminadas a lograr cambios perdurables en las relaciones de género, la OPS utiliza un instrumento de planificación de abordajes prácticos y estratégicos de género (13).

El abordaje práctico de género (APG) está diseñado para dar respuestas inmediatas a las necesidades de salud particulares de hombres y mujeres, sin intentar corregir a fondo las inequidades de género subyacentes. He aquí un ejemplo: A una mujer que vive en una situación de violencia doméstica sexual se le proporcionan anticonceptivos sin el consentimiento de su pareja. Como es evidente, este APG satisface la necesidad particular de la mujer de prevenir un embarazo no deseado, pero plantea el peligro de reforzar o incluso exacerbar las inequidades de género existentes. Con el fin de evitar consecuencias de este tipo, la OPS promueve el llamado abordaje estratégico de género (AEG), que no solo responde a las necesidades concretas de las mujeres y los hombres, sino que se orienta a redistribuir el poder entre ellos, así como sus respectivos papeles y responsabilidades. A la medida descrita en el ejemplo se sumaría la remisión de la mujer por los servicios de salud reproductiva a alguna entidad con la capacidad para ayudarla a salir de su mala situación doméstica, o la remisión del cónyuge a sesiones de terapia para hombres agresivos, con el consentimiento de su mujer.

Tanto el APG como el AEG reflejan la conciencia de que hay inequidades en función del género, pero uno busca corregirlas en el plazo inmediato y el otro en el largo plazo. La aplicación de estos abordajes con el fin de promover la participación de los hombres en los programas de salud reproductiva puede facilitar la definición de objetivos claros y mensurables en el mediano y largo plazo. Asimismo, sirve para construir puentes entre quienes opinan a favor y en contra de tratar los temas de la masculinidad y las faltas de equidad de género en el contexto de los programas de salud reproductiva.

\section{LOS DERECHOS SEXUALES Y REPRODUCTIVOS DE HOMBRES Y MUJERES}

Hoy en día también se debate la relación entre los derechos sexuales y reproductivos ${ }^{6}$ de los hombres y los de las mujeres. La perspectiva de género guarda estrecha relación con el tema de los derechos humanos. La abogacía en el área de la salud sexual y reproductiva ha solido centrarse en los derechos de las mujeres como grupo social en desventaja, pero actualmente, la necesidad de definir el papel de los hombres en la salud sexual y reproductiva exige un análisis de la relevancia y pertinencia de promover sus derechos sexuales y reproductivos, proceso que se encuentra en etapa incipiente.

Hay opiniones a favor y en contra de la perspectiva de equidad de género. Aunque para la mayoría se trata de un componente intrínseco de los derechos sexuales y reproductivos, algunas agrupaciones de hombres defienden el ejercicio de los derechos masculinos absolutos, pasando por encima de los derechos de las mujeres, porque se aferran a un concepto hegemónico y poco equitativo de la masculinidad.

En el plano individual, los derechos sexuales y reproductivos, tan estrechamente vinculados con los derechos humanos, se aplican a cada persona, sea mujer $u$ hombre. Pero algunos autores consideran que el concepto de derechos sexuales y reproductivos no se aplica a los hombres, ya que, como grupo social homogéneo, los hombres no sufren la misma discriminación y falta de equidad que las mujeres (21). El Centro de Investigación Social, Tecnología Apropiada y Capacitación (CISTAC), ONG dedicada a la equidad de género, señala algunos problemas que han surgido en su trabajo con los hombres en Bolivia: "Cuando CISTAC preguntó a los hombres qué significaba para ellos el concepto de 'derechos sexuales y reproductivos', tendían a ver aspectos de la masculinidad tradicional como derechos 'naturalizados'. Para trabajar el concepto de derechos con hombres, CISTAC recomienda primero 'deconstruir' y luego 'reconstruir' sus nociones de derechos, para que así incluyan los derechos de las mujeres (14)".

\footnotetext{
6 La plataforma de acción de Beijing define así los derechos sexuales y reproductivos: "Esos derechos se basan en el reconocimiento del derecho básico de todas las parejas e individuos a decidir libre y responsablemente el número de hijos, el espacionamiento de los nacimientos y el intervalo entre éstos y a disponer de la información y de los medios para ello y el derecho a alcanzar el nivel más elevado de salud sexual y reproductiva. También incluye su derecho a adoptar decisiones relativas a la reproducción sin sufrir discriminación, coacciones ni violencia, de conformidad con lo establecido en los documentos de derechos humanos" (20).
} 
Para evitar que la promoción de los derechos sexuales y reproductivos de los hombres entren en conflicto con el ejerecicio de los derechos de las mujeres, Benno de Keijzer (19) sostiene que existe la responsabilidad de respetar el mismo derecho o su equivalente tanto en el hombre como en la mujer. Varias organizaciones defienden los derechos de las mujeres cuando estos se ven amenazados por los derechos de los hombres, aduciendo las inequidades de género que sufren las mujeres como grupo social (14).

A pesar de que los hombres, en general, no enfrentan las mismas situaciones de desventaja que las mujeres, las diversas masculinidades que se suman a la masculinidad hegemónica llevan a tener presentes las inequidades que pueden sufrir grupos particulares, como los niños, los hombres jóvenes, y los homosexuales e indígenas (22). El concepto central de los derechos sexuales y reproductivos - que cada persona pueda tomar decisiones libremente en materia de sexualidad y reproducción-claramente guarda relación con la defensa de los derechos de grupos particulares.

La definición de los derechos sexuales y reproductivos de los hombres genera ciertos dilemas. Por ejemplo, ¿cómo se puede reconciliar el derecho de un hombre a ser padre con el derecho de su pareja a no ser madre? En este caso, muchos probablemente argumentan que la mujer tiene derecho a tomar decisiones relacionadas con su propio cuerpo. ¿Pero qué ocurriría si la situación fuera al revés? ¿Cómo definir el derecho de un hombre a negarse a la paternidad biológica o social? Las nuevas tecnologías que facilitan la reproducción ponen de relieve la importancia de estos dilemas éticos en torno a los derechos reproductivos de ambos sexos y su mutua compatibilidad.

Es necesario reflexionar más detenidamente sobre los derechos particulares de los hombres desde el punto de vista de la ética, la equidad de género y los derechos humanos en general. En este proceso, la participación de las mujeres es imprescindible para evitar poner en peligro sus propios derechos sexuales y reproductivos.

\section{SEPARACIÓN O INTEGRACIÓN DE SERVICIOS DE SALUD REPRODUCTIVA PARA HOMBRES Y MUJERES}

Un último debate que se aborda en este artículo guarda relación con la idoneidad de crear servicios de salud sexual y reproductiva separados para hombres, o de integrarlos con los programas ya existentes. En América Latina se han creado algunos programas y servicios de salud reproductiva exclusivamente para hombres, con diferentes resultados y grado de sostenibilidad $(14,15)$. Quienes abogan por incluir a los hombres en los servicios existentes ponen en tela de juicio el valor de estas iniciativas, bajo el argumento de que se pierden oportunidades para mejorar la comunicación y la toma de decisiones conjuntas entre los hombres y sus parejas heterosexuales (23).

Por otro lado, incluir a los hombres en servicios de salud que han sido establecidos para mujeres entraña diversas dificultades. Según han demostrado varias investigaciones dentro y fuera de América Latina, los hombres no confían en que los servicios de salud reproductiva, a los cuales identifican con la atención maternoinfantil, puedan responder a sus necesidades. Muchos no acuden a dichos servicios porque se preocupan menos que las mujeres por la salud reproductiva. Actualmente, los servicios de salud pública en América Latina no cuentan con personal capacitado ni con las herramientas necesarias para atender a las necesidades particulares de los hombres. Tampoco existen políticas institucionales o normas de atención adaptadas a las necesidades de la población masculina $(1$, $8,14,24)$.

Independientemente de que los servicios para hombres y mujeres se mantengan separados o se integren, siguen siendo objeto de debate los componentes clave que deben ser incorporados en los programas de salud reproductiva para responder a las necesidades específicas de los hombres. Los siguientes son apenas los más elementales: planificación familiar, incluida la vasectomía; atención de la infertilidad; prevención y tratamiento de las infecciones de transmisión sexual, entre ellas la infección por VIH y el sida; la sexualidad y sus trastornos; afecciones urológicas; cáncer; abuso de sustancias; salud mental; prevención de la violencia general y de género; y promoción de actitudes y prácticas responsables hacia la sexualidad y el embarazo, la paternidad y la crianza de los hijos (25).

Los procesos de reforma en la Región, cuyos elementos clave son la descentralización, la privatización y el mejoramiento de la accesibilidad, calidad y eficiencia de los servicios en función del costo, pueden ofrecer un contexto adecuado para prestar la debida atención a las necesidades de los hombres. Sin embargo, se interponen algunos obstáculos. Uno de ellos es la necesidad, impuesta por la escasez de recursos, de sopesar la efectividad y el costo de los programas. Otro es que las pocas pruebas reunidas hasta ahora de la eficacia de incluir a los hombres en actividades de salud reproductiva se han basado en experiencias piloto de alcance limitado. Todo ello dificulta las negociaciones encaminadas a adquirir fondos especiales o adicionales para incluir a los hombres en esas actividades.

Por otra parte, los procesos de reforma del sector de la salud en América Latina no se orientan, en general, a lograr modelos de atención basados en la promoción de la salud. Dada la estrecha relación 
práctica y conceptual entre la promoción de la salud y la lucha contra las inequidades de género en materia de sexualidad y reproducción, esta situación podría menoscabar la inclusión de los hombres en los programas de salud reproductiva.

Se ha demostrado, mediante algunas iniciativas piloto, que incorporar la atención de los hombres en los servicios de salud reproductiva no siempre exige grandes inversiones financieras. Se pueden aplicar medidas relativamente sencillas y baratas para lograr que los hombres acudan a estos servicios: establecer horas especiales de atención para ellos; invitarlos a concurrir con sus parejas; proporcionarles una entrada y salas de espera separadas; contratar a proveedores y educadores de sexo masculino, y modificar la decoración de la clínica (26). Asimismo, las actividades de promoción de la salud y de prevención también se pueden llevar a cabo fuera de los servicios de salud en instalaciones educativas, laborales o de recreo. Sea como fuere, la incorporación de la atención de los hombres en los programas públicos de salud reproductiva no es factible sin la disponibilidad de fondos presupuestarios, lo cual implica determinar primero qué inversiones serían las más efectivas en función del costo en el corto, mediano y largo plazo.

\section{LA PROMOCIÓN DE LA SALUD REPRODUCTIVA DEL HOMBRE Y LA MUJER}

Connell (12) señala que los hombres, a diferencia de las mujeres, no tienen un interés estructurado y compartido por cambiar las relaciones de género porque no se enfrentan a situaciones de inequidad como grupo social. El autor concluye que esto dificulta la creación de grupos de abogacía y presión al estilo de los movimientos internacionales y regionales que promueven la salud y los derechos de la mujer, como la Red de Salud de las Mujeres Latinoamericanas y del Caribe.

Pese a lo antedicho, en varios países de América Latina se han generado pequeños grupos de hombres que se dedican a asuntos relacionados con la masculinidad, la paternidad y la sexualidad. Algunos grupos de hombres en la Región han obtenido resultados alentadores en su lucha contra la violencia familiar. La mayoría de ellos tienen por meta luchar por la equidad de género, aunque simultáneamente están surgiendo otros grupos de hombres que defienden las relaciones de género tradicionales.

El contexto del ciclo vital en su totalidad ofrece grandes oportunidades para fomentar en los hombres actitudes y prácticas sanas y responsables en torno al cuidado de su propia salud, desde la niñez y a lo largo de las diferentes etapas de la vida. La Fundación Mexicana para la Planeación Familiar (MEXFAM) ha observado, por ejemplo, que los hombres más jóvenes ya muestran mayor interés en la equidad de género que los que pertenecen a generaciones anteriores. Señala, sin embargo, que estos jóvenes a menudo carecen de nuevos modelos masculinos, es decir, que el antiguo concepto de la masculinidad aún no ha sido reemplazado por uno nuevo (14).

Los debates actuales en América Latina y en el resto del mundo sobre la inclusión de los hombres en los servicios de salud reproductiva son intensos y a la vez sumamente interesantes. Para la OPS, este es un momento oportuno para prestar cooperación técnica destinada a promover una mayor equidad de género en la atención de la salud sexual y reproductiva en toda la Región de las Américas, dentro del contexto de actividades para la promoción de la salud. La promoción de la salud y de los derechos sexuales y reproductivos de hombres y mujeres a lo largo del ciclo vital les permitirá desarrollar habilidades para llevar una vida sexual y reproductiva sana y responsable y para decidir cuándo y con quién tener hijos sin coacción, discriminación o violencia y con respeto por la integridad física y mental de la pareja y toda la familia.

Agradecimiento. Se agradece el apoyo de David Acurio, antiguo residente del Programa sobre Mujer, Salud y Desarrollo, quien ha aportado su visión crítica durante la elaboración de este documento.

\section{SYNOPSIS}

\section{Men's participation in reproductive health programs: current debates in Latin America}

This article discusses current issues and debates surrounding the promotion of men's participation in public reproductive health programs in Latin America. There is little disagreement in the Region about the importance of this goal, but opinions differ as to how they should participate. The following topics are discussed in this article: the relationship between men's own reproductive health needs and their role in improving the reproductive health of their female partners; the need to analyze gender inequities and hegemonic masculinity; the relationship between men's and women's sexual and reproductive rights; and the need for separate services for men versus their integration in existing reproductive health services. The article concludes by underlining the importance of health promotion strategies throughout the life cycle in order to improve sustainable changes toward greater equity in reproductive health, within a genderoriented, ethical and human rights framework 


\section{REFERENCIAS}

1. Drennan M. Reproductive health: new perspectives on men's participation. Pop Rep 1998; Series J(46).

2. Organización Panamericana de la Salud Comité Ejecutivo del Consejo Directivo. Población y salud reproductiva. Washington, D.C.: OPS; 1998. (Documento CE122/11)

3. Berer M. Men. En: Reproductive Health Matters 1996;(7):7-10.

4. Organización Panamericana de la Salud Fondo de Población de las Naciones Unidas. La contribución de las ONGs a la salud y los derechos sexuales y reproductivos en América Latina. Recomendaciones de la sociedad civil para mejorar el seguimiento de los acuerdos de El Cairo y Beijing. Washington, D.C. FNUAP, OPS y RSMLAC; 1998.

5. World Health Organization. DALYs and reproductive health: report of an informal consultation, 1998. Geneva: WHO; 1999. (Documento WHO/RHT/98.28).

6. Organización Panamericana de la Salud. Bibliografía: hombres y diferencias de género en salud. Washington, D.C. OPS; 1999.

7. Universidade de São Paulo, Faculdade de Saúde Pública, Centro Colaborador da OMS par a Classificação do Doenças em Português. Perfil epidemiológico da saúde masculina na região das Americas: uma contribuição para o enfoque de gênero. São Paulo: Universidade de São Paulo; 1998

8. AVSC International, International Planned Parenthood Federation, Western Hemisphere Region. Resumen de publicaciones para el simposio sobre participación masculina en la salud sexual y reproductiva: nuevos paradigmas. New York: AVSC International \& IPPF/WHR 1998.

9. HIM. Helping involve men. An essential library on men and reproductive health [base de datos en CD-ROM]. Baltimore: POPLINE Digital Services; 2000.

10. Whitehead M. Los conceptos y principios de la equidad en la salud. Washing- ton, D.C.: Organización Panamericana de la Salud; 1991.

11. Connell RW. La organización social de la masculinidad. En: Valdés J, Olavarría J, eds. Masculinidad/es: poder y crisis. Santiago, Chile: Isis Internacional; 1997. pp. 31-48.

12. Connell RW. El imperialismo y el cuerpo de los hombres. En: Valdés J, Olavarría J, eds. Masculinidades y equidad de género en América Latina. Santiago, Chile: FLACSO-CHILE; 1998. pp. 76-89.

13. Hartigan P, Gómez E, de Schutter M, da Silva J. Taller sobre Género, Salud y Desarrollo: guía para facilitadores. Washington, D.C.: Organización Panamericana de la Salud; 1997.

14. AVSC International, International Planned Parenthood Federation, Western Hemisphere Region. Cinco casos de estudio para el simposio sobre participación masculina en la salud sexual y reproductiva: nuevos paradigmas. Nueva York: AVSC International \& IPPF/WHR; 1998

15. AVSC International, International Planned Parenthood Federation, Western Hemisphere Region. Memorias del simposio sobre participación masculina en la salud sexual y reproductiva: nuevos paradigmas. Nueva York: AVSC International \& IPPF/WHR; 1999

16. Gomensoro A, Lutz E, Güida C, Corsino D. Ser varón en el dos mil: la crisis del modelo tradicional de masculinidad y sus repercusiones. Montevideo: Gomensoro; 1998.

17. Valdés J, Olavarría J, eds. Masculinidades y equidad de género en América Latina. Santiago, Chile: FLACSO-CHILE; 1998.

18. Leñero Otero L. Los varones ante la planificación familiar. En: Elu MC, Langer A, eds. Maternidad sin riesgos en México. México, D.F.: IMES AC; 1994. pp. 141-151.

19. Keijzer B de. Los derechos sexuales y reproductivos desde la dimensión de la masculinidad. En: Figueroa CB. México diverso y desigual: enfoques socio- demográficos. México, D.F.: Colmex y Somede; 1999.

20. Naciones Unidas. Informe de la Cuarta Conferencia Mundial sobre la Mujer. Naciones Unidas (publicación en línea) 1995 (A/CONF.177/20) [citada 1996 Febrero 13]. Se encuentra en URL: gopher:// gopher.undp.org:70/00/unconfs/wom en/off/a-20.s

21. Steele Verme C, Wegner MN, Jerzowski $\mathrm{T}$. The language of male involvement: what do you mean by that? POPULI 1996:10-11.

22. Díaz AM, Gómez Alcaraz FH. Los derechos sexuales y reproductivos de los varones. Una reflexión acerca de la masculinidad y los derechos. Santa Fe de Bogotá: PROFAMILIA; 1998.

23. Laudari CA. Gender equity in reproductive and sexual health. UNFPA CST Workshop on male involvement in reproductive and sexual health programmes and services; November 9 to 13. Rome: Food and Agriculture Organization, World Health Organization, United Nations Fund for Population Activities; 1998

24. Ketting E. Men and their sexual and reproductive health: planned parenthood challenges (publicación periódica en línea); 1996. [citada 1998 abril 9];(2). Se encuentra en: URL:http://www.ippf. org/pubs/ppc/96_2/health.htm

25. Food and Agriculture Organization World Health Organization, United $\mathrm{Na}-$ tions Educational, Scientific, and Cultural Organization, United Nations Fund for Population Activities. Technical support services system. Male involvement in reproductive health: incorporating gender throughout the life cycle. (Occasional Papers Series 1998, 1).

26. Khorram S, Wells E. Involving men in reproductive health. Outlook 1997;14(3): $1-6$. 